\title{
Elliptic flow of thermal dileptons in relativistic nuclear collisions
}

\author{
Rupa Chatterjee, ${ }^{1}$ Dinesh K. Srivastava, ${ }^{1}$ Ulrich Heinz,${ }^{2}$ and Charles Gale ${ }^{3}$ \\ ${ }^{1}$ Variable Energy Cyclotron Centre, I/AF Bidhan Nagar, Kolkata 700 064, India \\ ${ }^{2}$ Physics Department, The Ohio State University, Columbus, Ohio 43210, USA \\ ${ }^{3}$ Department of Physics, McGill University, 3600 University Street, Montréal, H3A 2T8, Canada
}

(Received 13 February 2007; published 18 May 2007)

\begin{abstract}
We calculate the transverse momentum and invariant mass dependence of elliptic flow of thermal dileptons for $\mathrm{Au}+\mathrm{Au}$ collisions at the Relativistic Heavy Ion Collider. The system is described using hydrodynamics, with the assumption of formation of a thermalized quark-gluon plasma at some early time, followed by cooling through expansion, hadronization, and decoupling. Dileptons are emitted throughout the expansion history: by annihilation of quarks and antiquarks in the early quark-gluon plasma stage and through a set of hadronic reactions during the late hadronic stage. The resulting differential elliptic flow exhibits a rich structure, with different dilepton mass windows providing access to different stages of the expansion history. Elliptic flow measurements for dileptons, combined with those of hadrons and direct photons, are a powerful tool for mapping the time evolution of heavy-ion collisions.
\end{abstract}

DOI: 10.1103/PhysRevC.75.054909

PACS number(s): 25.75.-q, 12.38.Mh

\section{INTRODUCTION}

Evidence has been mounting for some time that collisions between heavy nuclei at ultrarelativistic energies lead to formation of quark-gluon plasma (QGP). Strong confirmations arise from recent observations at the Relativistic Heavy Ion Collider (RHIC) of large anisotropic flow of all hadronic species [1-4] and of a suppression of high- $p_{T}$ hadrons due to parton energy loss in the dense medium $[5,6]$. Signatures of direct photon emission [7-10], as well as preliminary results on (excess) dilepton production in such collisions, indicative of a hot early state, have also started emerging [11].

The radial and elliptic flow of hadrons observed in relativistic heavy-ion collisions at RHIC strongly suggest that the QGP created in these collisions acts like a strongly coupled plasma with almost perfect liquid behavior [12]. This conclusion is based on the successful prediction of the hadron momentum distributions, in particular of their anisotropies in noncentral collisions, by dynamical calculations that treat the expanding QGP as an ideal fluid [3,4,13-16]. Although no other equally successful model exists, one has to remain conscious of the fact that the new "perfect liquidity" paradigm is based on a model back-extrapolation of the measured data to the early stages of the collision that are not directly accessible with hadronic observables. There are strong arguments that this back-extrapolation is fairly unique [12] and hence that the above-mentioned qualitative conclusion is robust. On a quantitative level, however, the extraction from experimental data of the (small) QGP viscosity is presently hampered not only by the unavailability of consistent hydrodynamic codes for viscous relativistic fluids, but even more by uncertainties about the hydrodynamic effects of changes in the equation of state of the QGP matter [17] and about details of the initial conditions at the beginning of the hydrodynamic expansion stage $[13,18]$.

The next generation of RHIC experiments will aim at obtaining more quantitative information about the properties of the QGP and the subsequent hot hadronic matter, as well as on the process of hadronization itself. Although more precise hadronic flow measurements, in particular of elliptic flow in noncentral collisions, will further constrain the hydrodynamic evolution models and the QGP equation of state, it will be invaluable to have additional measurements on electromagnetic radiation, i.e., direct photons and dileptons that are penetrating probes [19], and access more directly the very early expansion stages [20]. Elliptic flow is generated very early, via the transformation of the initial spatial eccentricity of the nuclear overlap region into momentum anisotropies through the action of azimuthally anisotropic pressure gradients. With the passage of time, the pressure gradients equalize, and the growth of elliptic flow shuts itself off [21]. Photons and dileptons, which escape from the expanding fireball without reinteraction, will be able to probe specifically this early stage where the flow anisotropy first develops. Measurements of the elliptic flow of thermal photons and lepton pairs could thus provide particularly clean constraints on the QGP equation of state, with fewer ambiguities arising from theoretical uncertainties about the hadronization process and late-stage hadronic rescattering dynamics than in the case of hadronic flow measurements [16].

The azimuthal asymmetry of photons from jet-plasma interactions has been examined recently [22], and so has the elliptic flow of thermal photons [23], calculated with a hydrodynamic model for the expansion of the collision fireball. Contrary to the monotonic rise with $p_{T}$ of the hydrodynamically predicted differential elliptic flow $v_{2}\left(p_{T}\right)$ of hadrons, the thermal photon elliptic flow was shown to peak at transverse momenta of $1-2 \mathrm{GeV} / c$, decreasing at higher $p_{T}$ as a reflection of the decreasing hydrodynamic flow anisotropies as one goes backward in the expansion history of the collision fireball. In addition, the photon elliptic flow was seen to exhibit an interesting peak-valley structure at low transverse momenta, $p_{T} \sim 0.4-0.5 \mathrm{GeV} / c$, reflecting the dominance of different types of hadronic photon production mechanisms below and above this $p_{T}$ value [23]. In the present 
article we complement the work of Ref. [23] with a study of thermal dilepton (i.e., virtual photon) elliptic flow, where the invariant mass of the lepton pair (photon virtuality) provides an additional continuously tunable parameter. As we will show, the combined dependences of the elliptic flow of dileptons on their transverse momentum $p_{T}$ and invariant mass $M$ provide a very rich landscape of structures that can be used to set observational windows on specific stages of the fireball expansion. Future studies will further complement the analysis presented here by directly measuring the space-time structure of the early collision fireball with two-photon correlations. By combining the momentum structure of the (virtual) photon emitting source from flow measurements with its space-time structure from Hanbury Brown-Twiss correlations one should be able to provide a complete experimental characterization of the phase-space structure of the QGP during the early stages of the fireball expansion.

\section{DILEPTON EMISSION RATES AND SPECTRA}

Dileptons, like photons, are emitted from every stage of a heavy-ion collision, from the pre-equilibrium stage [24], the quark-gluon fluid [25-32], and the late hadronic matter [33-38]. An interesting outcome of dilepton emission studies has been the confirmation of medium modifications of the spectral properties of $\rho$ mesons $[37,39]$. Correlated charm and bottom decay provide another important source of dileptons, which will help estimate energy loss and the elliptic flow of heavy quarks [40]. It may be possible in principle to isolate them by determination of the decay vertices.

In the present article we focus on thermal emission of dileptons from the QGP and hadronic phases in a collectively expanding fireball. The dilepton spectrum is obtained [34] by integrating the thermal emission rate over the space-time history of the system. As a result, high- $M$ dileptons arise mostly from the hot early stage where hydrodynamic flow is weak but the spatial eccentricity of the source is large, whereas dileptons of lower mass are emitted when the temperature is low, the flow is strong and anisotropic, but the spatial eccentricity of the fireball has mostly disappeared. These generic expectations are, however, modulated by the fact that dilepton emission from the late hadronic phase is characterized by strong vector meson resonance peaks in the dilepton mass spectrum, which leads to strong variations of the relative weight of dilepton emission from the QGP and hadron gas phases on and off these resonances. This leads to very strong and interesting structures in the $p_{T}$-integrated elliptic flow $v_{2}(M)$ as a function of dilepton mass, which can be exploited for differential fireball microscopy.

The dilepton momentum spectrum can be written as

$$
\begin{aligned}
E \frac{d N_{\ell \bar{\ell}}}{d M^{2} d^{3} p} & =\frac{d N_{\ell \bar{\ell}}}{d M^{2} p_{T} d p_{T} d y d \phi} \\
& =\int\{(\ldots) \exp [-p \cdot u(x) / T(x)]\} d^{4} x,
\end{aligned}
$$

where the quantity inside the curly braces indicates the thermal emission rates from the QGP or hadronic matter, with the thermal Boltzmann factor extracted for emphasis.
We parametrize the dilepton (virtual photon) four-momentum as

$$
p^{\mu}=\left(M_{T} \cosh Y, p_{T} \cos \phi, p_{T} \sin \phi, M_{T} \sinh Y\right)
$$

and the flow velocity of the fireball fluid $u^{\mu}$ as

$$
u^{\mu}=\gamma_{T}\left[\cosh \eta, v_{x}(x, y, \tau), v_{y}(x, y, \tau), \sinh \eta\right]
$$

with

$$
\gamma_{T}=\left(1-v_{T}^{2}\right)^{-1 / 2}, \quad v_{T}^{2}=v_{x}^{2}+v_{y}^{2} .
$$

(We assume boost-invariant longitudinal expansion [41].) We use coordinates $\tau, x, y, \eta$ with volume element $d^{4} x=$ $\tau d \tau d x d y d \eta$, where $\tau=\left(t^{2}-z^{2}\right)^{1 / 2}$ is the longitudinal proper time and $\eta=\tanh ^{-1}(z / t)$ is the space-time rapidity. The dilepton momentum is parametrized by its rapidity $Y$, its transverse momentum $p_{T}=\left(p_{x}^{2}+p_{y}^{2}\right)^{1 / 2}$, and its azimuthal emission angle $\phi$.

The dilepton energy in the local fluid rest frame, which enters the Boltzmann factor in the thermal emission rate in the combination $p \cdot u / T$, is thus given by

$$
p \cdot u=\gamma_{T}\left[M_{T} \cosh (Y-\eta)-p_{T} v_{T} \cos \left(\phi-\phi_{v}\right)\right],
$$

where $M_{T}=\left(M^{2}+p_{T}^{2}\right)^{1 / 2}$ is the transverse mass and $M$ the invariant mass of the dilepton, and $\phi_{v}=\tan ^{-1}\left(v_{y} / v_{x}\right)$ is the azimuthal angle of the transverse flow vector. This expression shows that the azimuthal anisotropy ( $\phi$ dependence) of the dilepton spectrum, conventionally characterized by its Fourier coefficients $v_{n}$ (where only even $n$ contribute at $Y=0$ ),

$$
\begin{aligned}
\frac{d N(b)}{d M^{2} d^{2} p_{T} d Y}= & \frac{d N(b)}{d M^{2} 2 \pi p_{T} d p_{T} d Y} \\
& \times\left[1+2 v_{2}\left(M, p_{T}, b\right) \cos (2 \phi)+\cdots\right],
\end{aligned}
$$

is controlled by an interplay between the collective flow anisotropy and the geometric deformation of the temperature field $T(x, y, \tau)$ (see Ref. [23] for more details). It vanishes in the absence of radial flow, $v_{T}=0$.

We use the boost invariant hydrodynamic code AZHYDRO [42], which has been used extensively and successfully to describe midrapidity hadron production at RHIC and more recently to predict the elliptic flow of thermal photons [23]. We study $\mathrm{Au}+\mathrm{Au}$ collisions at $\sqrt{s}=200 \mathrm{~A} \mathrm{GeV}$ and use the same initial conditions as in Ref. [23], namely an initial time $\tau_{0}=0.2 \mathrm{fm} / c$ for the beginning of the hydrodynamic stage, with an initial peak entropy density in central collisions of $s_{0}=351 \mathrm{fm}^{-3}$, corresponding to a peak initial temperature of $T_{0}=520 \mathrm{MeV}$. Although the matter may not yet be completely thermalized at such an early time, we choose $\tau_{0}$ so small to also account at least partially for pre-equilibrium dilepton production at very early times $[24,43]$. This contribution to the dilepton spectrum is important at large $M$ and and large $p_{T}$, and it will suppress $v_{2}^{\ell \bar{\ell}}$ there because very little transverse flow develops before about $0.5 \mathrm{fm} / c$. The initial transverse entropy density profile is computed from a Glauber model assuming $75 \%$ wounded nucleon and $25 \%$ binary collision scaling of the initial entropy production [14]. This reproduces the collision centrality dependence of hadron production in $\mathrm{Au}+\mathrm{Au}$ collisions at RHIC [14,44]. 
We assume that a thermally and chemically equilibrated plasma is formed at the initial time, and use the Born term for the production of dileptons from the QGP. It is known $[30,31]$ that for thermal dileptons the corrections to the Born term are $\sim\left(2 \pi \alpha_{s} / 3\right) T^{2} / M^{2}$ and thus decrease rapidly with increasing dilepton mass. For the production of dileptons from the hadronic reactions, we consider the comprehensive set of hadronic reactions analyzed by Kvasnikova et al. [38], which are known to correctly reproduce the spectral density measured in $e^{+} e^{-}$scattering. In Ref. [38] these rates are conveniently parametrized in the form $F_{\text {eff }}(M) \exp (-E / T)$. They have been shown to provide a good description of intermediate mass dilepton data from $\mathrm{Pb}+\mathrm{Pb}$ collisions at the SPS [38]. However, because these rates are used here for lower invariant masses, some comments are in order. For instance, it is known that vector meson spectral densities are modified in the medium [19], and such effects are not included in the rates of Ref. [38]. Still, as long as they do not produce severe distortions of the transverse momentum spectra, the dilepton elliptic flow signal evaluated here should be relatively robust.

For the equation of state we use EOS Q [42], which matches a free QGP to a chemically equilibrated hadron resonance gas (HG) by a Maxwell construction at critical temperature $T_{c}=164 \mathrm{MeV}$, with energy densities $\epsilon_{\mathrm{q}}=$ $1.6 \mathrm{GeV} / \mathrm{fm}^{3}$ and $\epsilon_{\mathrm{h}}=0.45 \mathrm{GeV} / \mathrm{fm}^{3}$ in the QGP and HG subphases at this temperature. Hadron freeze-out is assumed to happen at $\epsilon_{\mathrm{f}}=0.075 \mathrm{GeV} / \mathrm{fm}^{3}[14]$.

Figure 1 shows the production of thermal dileptons from the quark matter and the hadronic matter for central collisions. Similar figures emerge at other impact parameters and help us understand the relative importance of hadronic and quarkmatter contributions at a given $M$. The QGP contribution (dotted line) is seen to completely dominate the mass spectrum of thermal dilepton radiation for dilepton masses above $1 \mathrm{GeV}$, except for the $\phi$ meson peak. Hadronic radiation (dashed line) dominates for $0.5 \mathrm{GeV}<M<1 \mathrm{GeV}$. The red dash-dotted lines show post-freeze-out decay contributions from $\rho, \omega$, and $\phi$ mesons; they are not included in the solid line for the total hydrodynamic spectrum. The sharply peaked solid black line

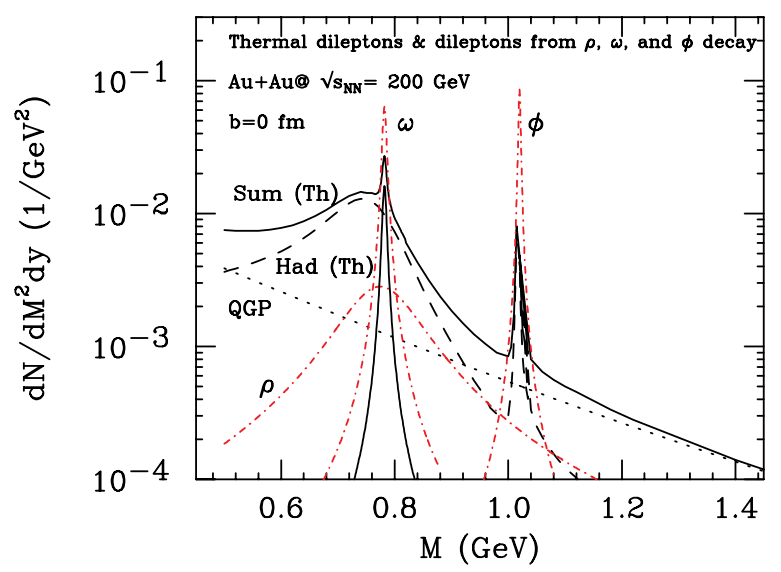

FIG. 1. (Color online) The mass spectrum of thermal dileptons from a hydrodynamical simulation of central $200 \mathrm{~A} \mathrm{GeV} \mathrm{Au+Au}$ collisions $(b=0)$. The quark and hadronic matter contributions are shown separately. See text for details. under the $\omega$ peak (which $i$ included in the total hydrodynamic spectrum) shows the contribution from thermally distributed $\omega$ mesons in the hadronic phase before freeze-out Because the hadronic dilepton emission rates given in Ref. [38] do not include three-body scattering channels proceeding through the $\omega$ meson, we include the $\omega$ explicitly as a thermally distributed particle species, with its vacuum spectral function, integrating its density multiplied with the standard partial width for dilepton decay over the space-time volume of the hadronic phase contained inside the hadronic freeze-out surface. We see that post-freeze-out decays of $\omega$ and $\phi$ produce many more dileptons at the respective vector meson peaks than collisions and decays before hadronic freeze-out; this reflects the relatively long lifetimes of these two vector mesons (23.4 and $44.5 \mathrm{fm} / c$, respectively).

We pause here for a moment to note that the relative contributions of the emission from the hadronic and quark matter stages control the overall $v_{2}$ for the dileptons. Thus we shall see in the following that the elliptic flow of dileptons with masses near those of the $\rho, \omega$, or $\phi$ mesons is decided by the $v_{2}$ of the radiation from the hadronic matter. The elliptic flow parameter for dileptons with $M \gg 1 \mathrm{GeV}$, however, is dominated by quark matter radiation (see Fig. 6). To measure this experimentally would obviously be very valuable.

In Figs. 2 and 3 we show the transverse momentum spectra and $p_{T}$-dependent elliptic flow $v_{2}\left(p_{T}\right)$ for dileptons with invariant masses $M=m_{\rho}$ (Fig. 2) and $M=m_{\phi}$ (Fig. 3) from semiperipheral $\mathrm{Au}+\mathrm{Au}$ collisions at impact parameter $b=$ $7 \mathrm{fm}$. The bottom panels of these figures show that the elliptic flow of hadronic dileptons, $v_{2}(\mathrm{HM})$, is large and basically agrees with the elliptic flow of hadrons of the same mass $\left(v_{2}(\rho)\right.$ and $v_{2}(\phi)$, respectively), which are emitted from the hadronic freeze-out surface. The somewhat smaller differential elliptic flow $v_{2}\left(p_{T}\right)$ for the hadrons (compared to that of dileptons with the same mass) arises from their somewhat flatter $p_{T}$ spectrum (not shown). Because the hadrons decouple later than the average hadronic dilepton, their spectra are boosted by somewhat larger radial flow, and flatter spectra result in smaller differential elliptic flow $v_{2}\left(p_{T}\right)$ (see last article in Ref. [3]). The elliptic flow of thermal dileptons from the QGP phase, $v_{2}(\mathrm{QM})$, is much smaller and shows the same decrease at large $p_{T}$ that we already observed for thermal photons [23]. It also decreases with increasing dilepton mass. These features reflect the small flow anisotropies during the early QGP stage, especially for dileptons with large $p_{T}$ and $M$ whose production requires high temperatures and is therefore peaked at very early times. One should keep in mind, however, that the present analysis does not include lepton pairs produced via the Drell-Yan and jet fragmentation processes, nor those produced through jet-plasma interactions [45]. These can contribute their own characteristic azimuthal asymmetries [22]. A consistent inclusion of these nonthermal contributions is part of a study in progress.

The total dilepton flow $v_{2}$ is seen to follow that of the hadronic dileptons, $v_{2}(\mathrm{HM})$, up to rather large transverse momenta of about $2 \mathrm{GeV} / c$, before peaking near $3 \mathrm{GeV} / c$ and then decreasing at even larger $p_{T}$. This is due to the strong dominance of hadronic dilepton emission near the vector meson peaks in the dilepton mass spectrum of 

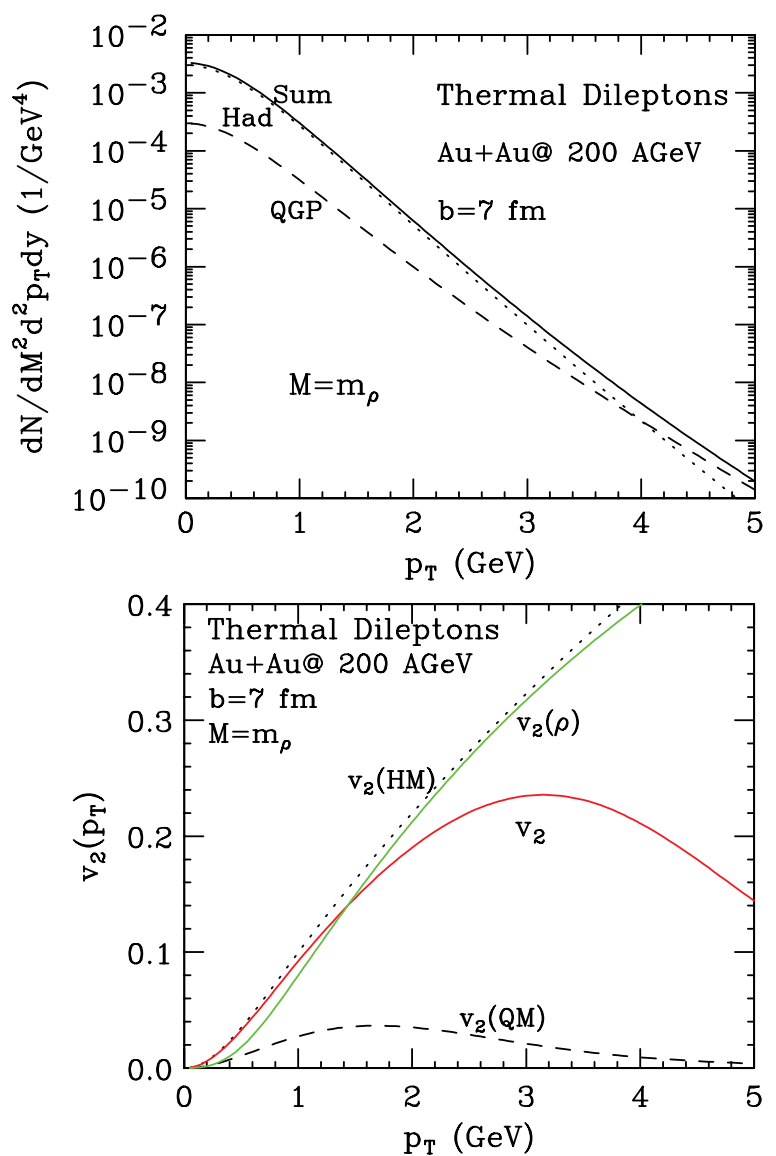

FIG. 2. (Color online) The transverse momentum spectrum (upper panel) and differential elliptic flow $v_{2}\left(p_{T}\right)$ (lower panel) of thermal dileptons with invariant mass $M=m_{\rho}$. Dashed and dotted lines show quark matter (QGP,QM) and hadron matter (Had,HM) contributions, respectively. In the bottom panel we also show for comparison the elliptic flow of $\rho$ mesons emitted from the hadronic decoupling surface.

Fig. 1: As seen in the top panels of Figs. 2 and 3, the dilepton $p_{T}$ spectrum is dominated by hadronic emission over almost the entire shown $p_{T}$ range, with QGP radiation winning out only for transverse momenta above $\sim 4 \mathrm{GeV} / c$. Note that a possible medium-induced suppression of the hadronic dilepton contribution by $\sim 50 \%$ near the $\rho$ mass would decrease the net $v_{2}$ there by only $10 \%$, because it is a weighted average. Still, the absence of nonthermal sources such as those enumerated at the end of the preceding paragraph must be kept in mind and should be corrected before attempting a quantitative comparison with future dilepton flow data.

In Fig. 4 we show that the success of the hydrodynamic model in describing all measured hadron $p_{T}$ spectra, at least up to $p_{T} \simeq 2 \mathrm{GeV} / c$, carries over to that of the $\phi$ meson, recently measured by the PHENIX Collaboration [46] via reconstruction of $\phi$ mesons from $K^{+} K^{-}$pairs. It can thus be hoped that the predicted spectrum and elliptic flow of dileptons with the same mass, as shown in Fig. 3, will be similarly reliable.

For dileptons with invariant mass $M=2 \mathrm{GeV}$ the $p_{T}$ spectrum is completely dominated by emission from the QGP
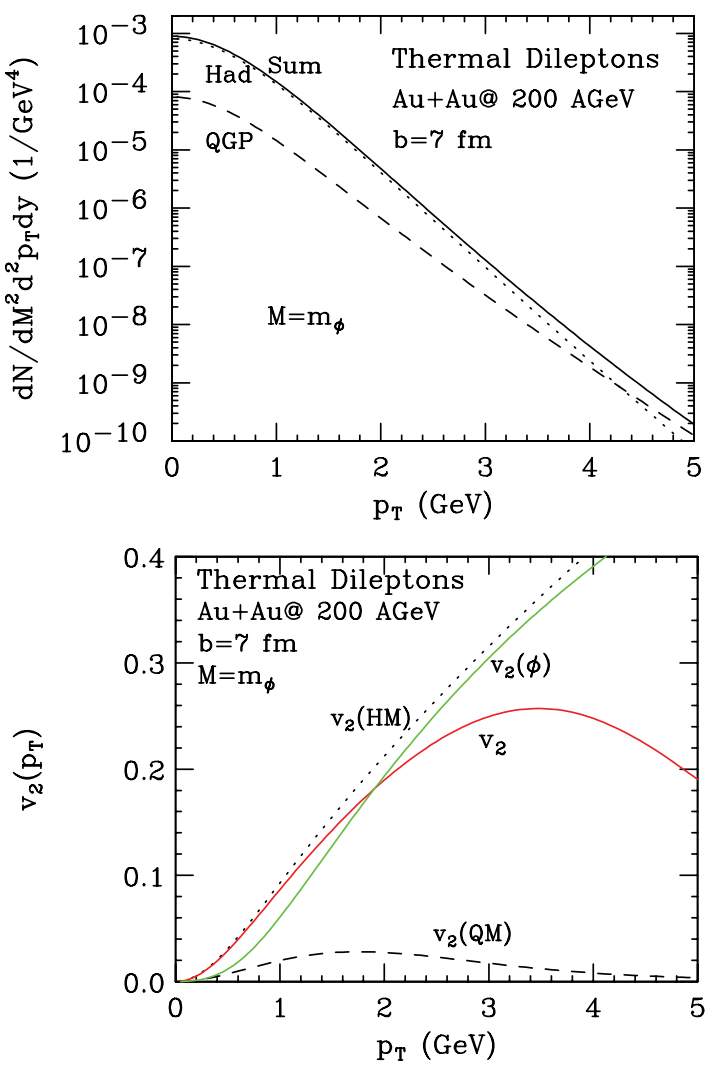

FIG. 3. (Color online) Same as Fig. 2, but for dileptons with invariant $M=m_{\phi}$, and with the elliptic flow of $\phi$ mesons from the hadronic decoupling surface shown in the bottom panel for comparison.

(Fig. 5, top panel). Correspondingly, the total dilepton elliptic flow $v_{2}$ closely follows that of the quark matter contribution, $v_{2}(\mathrm{QM})$, giving us a direct measurement of the flow anisotropy developed during the quark phase. Its decrease at high $p_{T}$ again reflects the decreasing flow anisotropies at earlier and earlier times. The total elliptic flow is small, peaking near $p_{T} \simeq$ $2 \mathrm{GeV} / c$ at a value of about $0.5 \%$, even though the elliptic

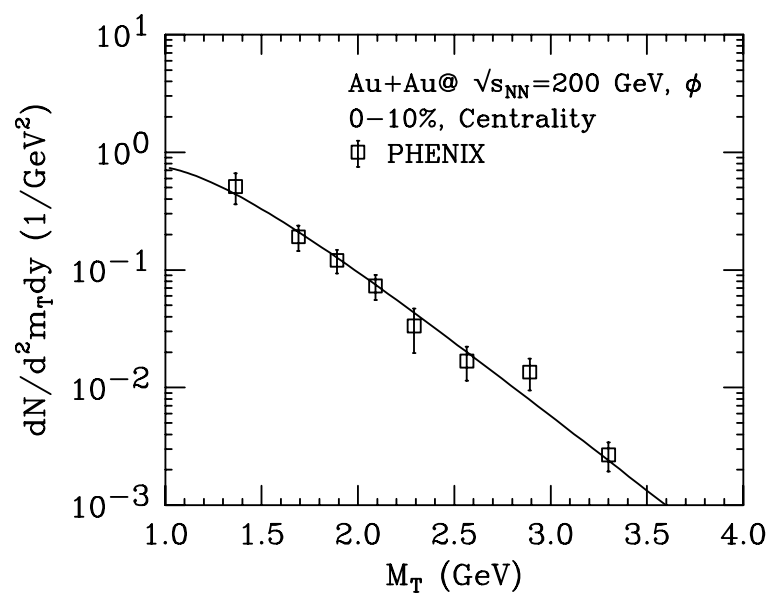

FIG. 4. Transverse momentum spectrum of $\phi$ mesons, comparing the theoretical prediction from the hydrodynamic model with PHENIX data Ref. [46]. 

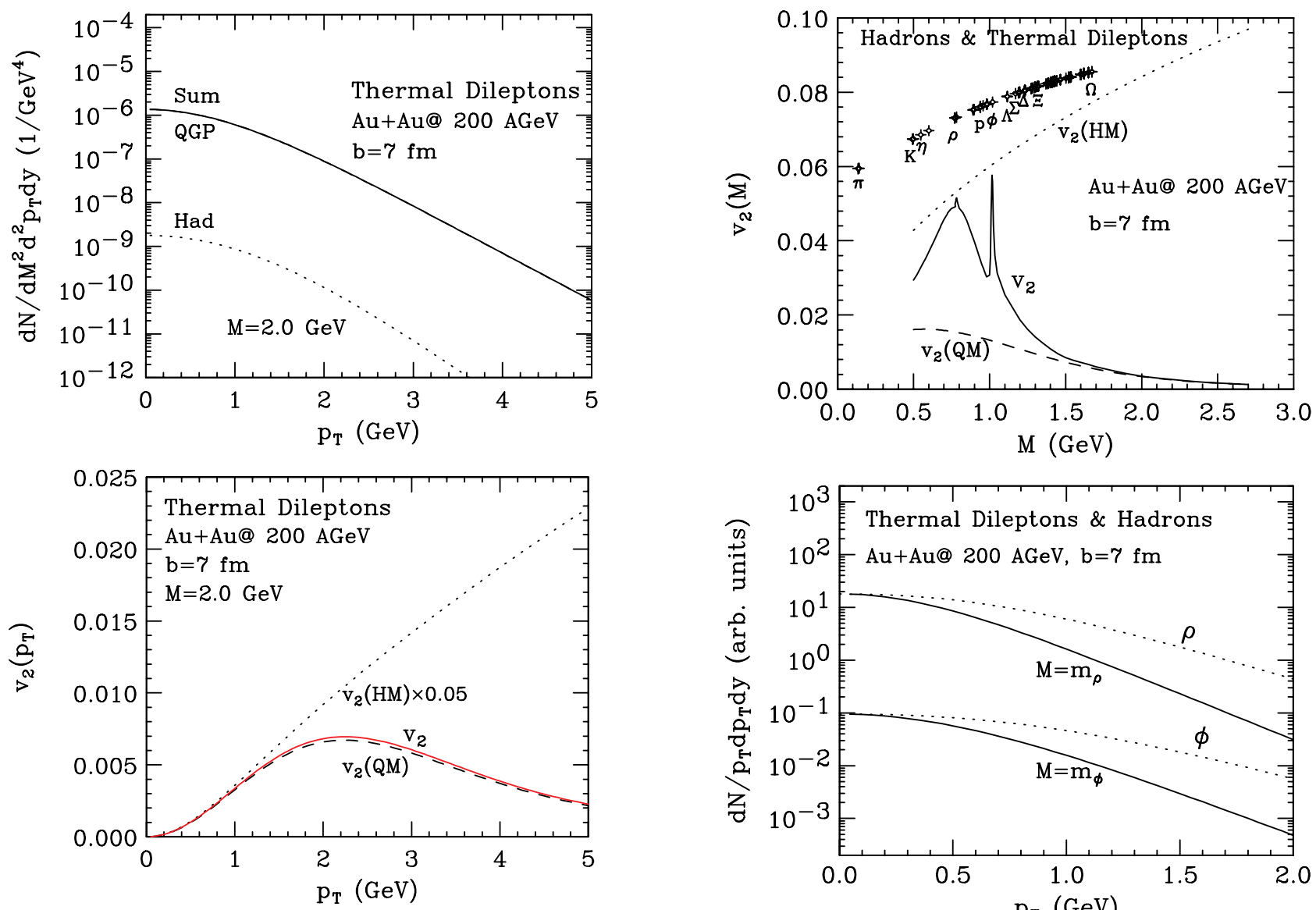

FIG. 5. (Color online) Same as Fig. 2, for $M=2 \mathrm{GeV}$.

flow of the (tiny) hadronic contribution of the same mass is about 20 times larger.

The upper panel of Fig. 6 shows the $p_{T}$-integrated elliptic flow as a function of dilepton mass, $v_{2}(M)$. The solid line gives the total elliptic flow of all dileptons (excluding, however, post-freeze-out decay dileptons), whereas the dashed and dotted lines show the elliptic flow of the QGP and hadronic dileptons separately. Note that the latter show similar qualitative behaviour as functions of $M$ and of $p_{T}$ : although the elliptic flow of hadronic dileptons increases monotonically with $M$ and $p_{T}$, the quark matter dileptons exhibit elliptic flow that first rises, then peaks, and finally decreases with increasing $M$ and/or $p_{T}$ [47]. For comparison, that panel also indicates the $p_{T}$-integrated elliptic flow values for a variety of stable hadron species and hadronic resonances emitted from the hadronic decoupling surface (see also the last paper in Ref. [3]). For large invariant masses the $p_{T}$-integrated elliptic flow of hadronic dileptons is seen to approach that of hadrons with the same mass. This reflects the fact that the homogeneity regions [50] for massive particles are small because their thermal wavelength decreases like $1 / \sqrt{M}$, and therefore all particles of that mass (hadrons or virtual photons) feel approximately the same flow. Low-mass hadronic dileptons, however, are emitted from much larger homogeneity regions and thus have an appreciable chance of being emitted significantly earlier than the corresponding hadrons of the same mass; on average they thus carry less radial and elliptic flow.
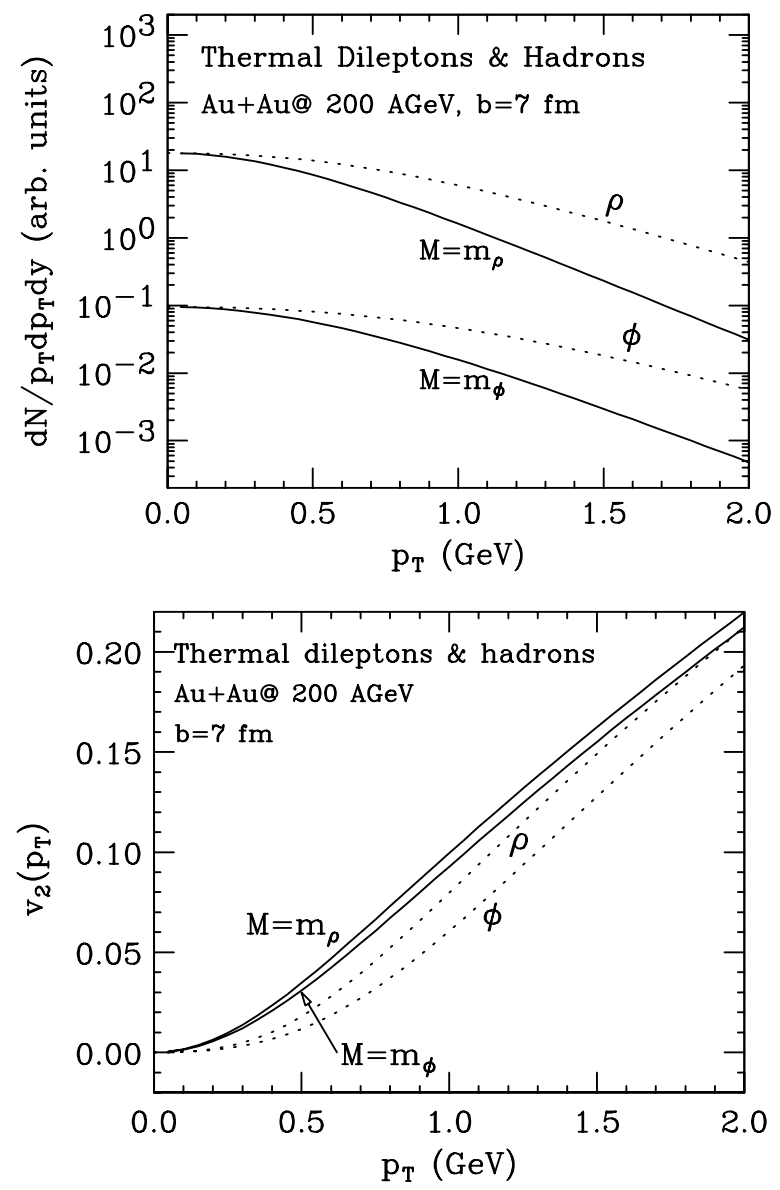

FIG. 6. (Upper panel) $p_{T}$-integrated elliptic flow parameter for dileptons and various hadrons. (Middle panel) $p_{T}$ distributions of dileptons with $M=m_{\phi}$ and $M=m_{\rho}$, and of $\phi$ and $\rho$ mesons. (Lower panel) Differential elliptic flow for the above.

We note that the additional buildup of radial and (to a lesser extent) elliptic flow during the hadronic stage has opposite effects on the $p_{T}$-integrated and $p_{T}$-differential elliptic flows: Although the $p_{T}$-integrated elliptic flow increases, the additional broadening of the single-particle spectrum from extra radial flow shifts the weight of the flow anisotropy to larger $p_{T}$, so at fixed $p_{T}$ the elliptic flow decreases (at least in the low- $p_{T}$ region where $v_{2}\left(p_{T}\right)$ is a rising function of $\left.p_{T}\right)$. This follows 

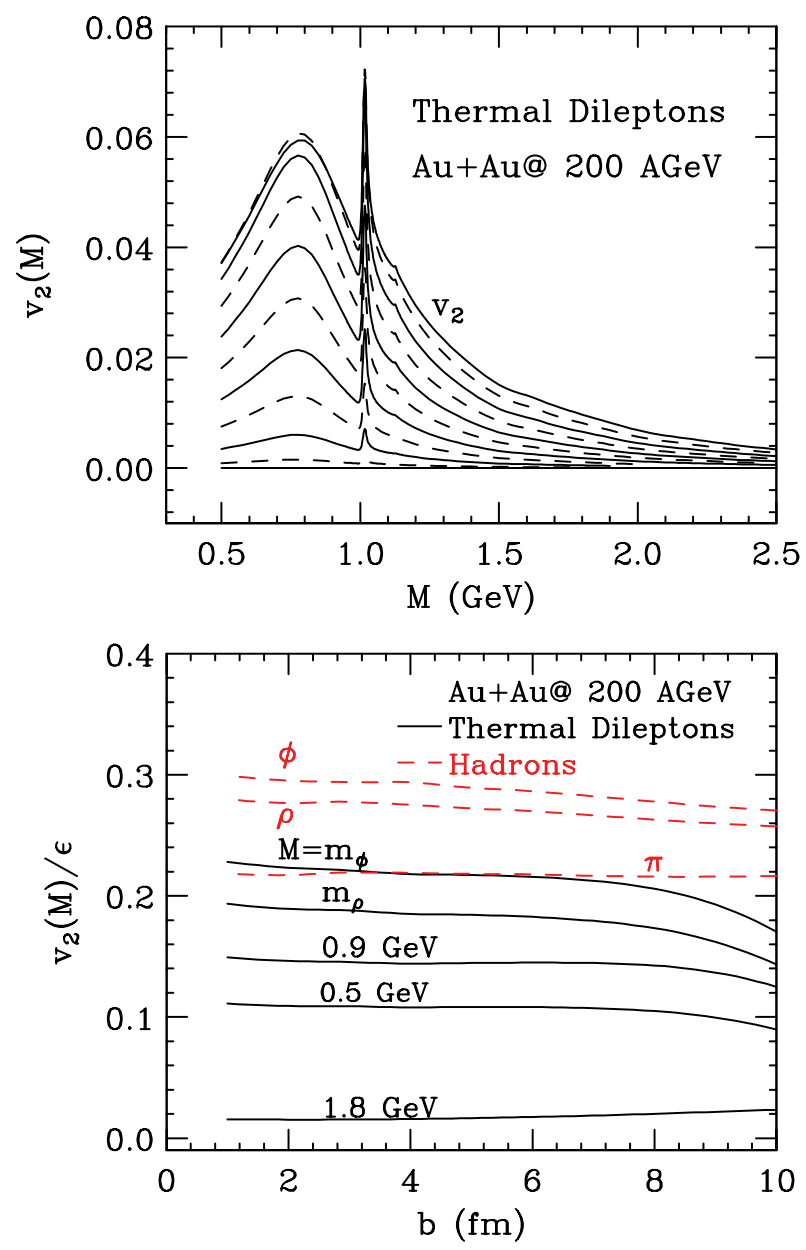

FIG. 7. (Color online) (Top panel) Dilepton mass dependence of the $p_{T}$-integrated elliptic flow parameter for thermal dileptons, for a variety of impact parameters $(b=0,2,4,6,8,10 \mathrm{fm}$ from bottom to top (solid curves) and $b=1,3,5,7,9 \mathrm{fm}$ (dashed curves)). Note that $v_{2}$ for $b=10 \mathrm{fm}$ is marginally smaller than for $b=9 \mathrm{fm}$. (Bottom panel) Impact parameter dependence of the $p_{T}$-integrated elliptic flow parameter scaled by the initial spatial eccentricity $\epsilon$, for a variety of hadrons and dileptons with different masses.

directly from the discussion presented in the last article of Ref. [3], and it is clearly seen in the figures given in the middle and the lower panels of Fig. 6. The middle panel shows the (arbitrarily normalized) $p_{T}$ distributions of dileptons having masses equal to $m_{\rho}$ and $m_{\phi}$, respectively, compared to those of the $\rho$ and $\phi$ mesons themselves. The hadron $p_{T}$-spectra are clearly seen to decrease more slowly with $p_{T}$, as stated above. (We stress that the continued hydrodynamical growth of radial flow during the hadronic matter stage is essential for this pattern; if one neglects transverse flow one arrives at the opposite conclusion [51].) The lower panel in Fig. 6 then demonstrates that this flow-induced flattening of the hadronic $p_{T}$ spectra leads to a systematic lowering of the differential elliptic flow $v_{2}\left(p_{T}\right)$ for these hadrons when compared to dileptons of similar masses. We shall see later in Fig. 7 (lower panel) that this is accompanied by larger values for the $p_{T}$-integrated flow paramters for $\rho$ and $\phi$ mesons compared to that of dileptons having similar masses.
As seen in the upper panel of Fig. 6, the elliptic flow of the total dilepton spectrum shows dramatic structure as a function of dilepton mass, oscillating between the quark and hadronic matter limits. This reflects the relative weight of the QM and HM contributions to the dilepton mass spectrum: Close to the vector meson resonance peaks, dilepton emission is strongly dominated by emission from the hadron matter phase, and the solid line almost reaches the dotted curve for $v_{2}(\mathrm{HM})$. At low dilepton masses, between the $\rho$ and $\phi$ mesons, and for dilepton masses $\gtrsim 1.5 \mathrm{GeV}$, quark matter radiation dominates the dilepton spectrum, resulting in small elliptic flow coefficients (of order $1 \%$ or less) for the total dilepton spectrum, consistent with the pure quark matter contribution $v_{2}(\mathrm{QM})$ (dashed line). By setting appropriate invariant mass windows on the dileptons one can thus peek into the early QGP phase and study the beginning of elliptic flow buildup.

Figure 7 shows the impact parameter dependence of the $p_{T}$-integrated elliptic flow parameter for thermal dileptons, as a function of dilepton mass $M$. The top panel shows that, as the impact parameter (and thus the spatial eccentricity $\epsilon=\left\langle y^{2}-x^{2}\right\rangle /\left\langle y^{2}+x^{2}\right\rangle$ of the initial nuclear overlap region) increases, the elliptic flow $v_{2}$ increases, too, as naively expected. In the bottom panel we therefore study the eccentricity-scaled elliptic flow, $v_{2} / \epsilon$, as a function of collision centrality, for several hadron species and dileptons with a variety of different masses. Even though the dileptons are, on average, emitted earlier (i.e., with less radial flow) than the hadrons, we see no dramatic differences between the impact parameter dependences of hadronic and dilepton $v_{2}$ : Both are almost independent of collision centrality, except for the small drop of $v_{2} / \epsilon$ for dileptons with masses $M \lesssim$ $1 \mathrm{GeV}$ at large impact parameters $b \gtrsim 8 \mathrm{fm}$. This reaffirms the general understanding $[3,52]$ that for ideal fluids the ratio $v_{2} / \epsilon$ reflects the effective stiffness (sound speed) of the fireball medium's equation of state, averaged over the expansion history. The changing weight between QGP and hadronic dilepton emission for different dilepton masses is seen to only affect the magnitude of their elliptic flow, but not its collision centrality (in-)dependence. (Note that the $\omega$ contribution was not included in Fig. 7. The also omitted Drell-Yan contribution is expected to emerge only at larger invariant masses than those shown in Fig. 7 [53].)

\section{CONCLUSIONS}

We have presented a first hydrodynamic calculation of elliptic flow of thermal dileptons emitted from ultrarelativistic heavy-ion collisions at RHIC energies. The azimuthal flow parameter exhibits a rich structure as a function of transverse momentum and invariant mass. When combined with photon elliptic flow measurements [23], this yields a new versatile and potentially very powerful probe of the fireball dynamics at RHIC and LHC, complementary to the already wellstudied flow anisotropies in the hadronic sector. $v_{2}^{\gamma}\left(p_{T}\right)$ and $v_{2}^{\ell \bar{\ell}}(M)$ exhibit rich structures that reflect the interplay of different emission processes, opening a window on detailed and differential information from a variety of different stages of the fireball expansion. The elliptic flow of photons emitted 
from the late hadronic stage was seen [23] to track the $v_{2}$ of the emitting hadrons, suggesting the possibility of subtracting the hadronic emission contributions from the total photon signal to isolate and study in greater detail the elliptic flow of early QGP photons. Similarly, we saw in the present study that at low $(M<0.5 \mathrm{GeV})$ and high $(M>1.5 \mathrm{GeV})$ invariant masses dilepton emission is completely dominated by emission from the early quark matter, without need for subtracting hadronic contributions. However, at low $M$ experimental backgrounds are large, whereas at high $M$ thermal dilepton yields are small. Clean elliptic flow measurements of the early QGP stage are therefore hard.

Obviously, when compared to hadrons, electromagnetic probes suffer from their relatively small production cross sections, so these measurements require dedication. However, the first glimpse offered here suggests that photon and dilepton elliptic flow have the potential of turning into powerful and highly discriminating tools for heavy-ion phenomenology and for differential studies of the fireball expansion. Extensive further theoretical investigations are therefore warranted, in particular with respect to the inclusion of viscous effects at intermediate and high transverse momenta.

\section{ACKNOWLEDGMENTS}

C.G. thanks Jörg Ruppert and Simon Turbide for useful conversations. U.H. acknowledges the hospitality of the Institute for Nuclear Theory at the University of Washington, where parts of this work were completed. The research reported here was supported in part by the U.S. Department of Energy under contract no. DE-FG02-01ER41190 and in part by the Natural Sciences and Engineering Research Council of Canada.
[1] C. Adler et al. (STAR Collaboration), Phys. Rev. Lett. 87, 182301 (2001); 89, 132301 (2002); K. Adcox et al. (PHENIX Collaboration), ibid. 89, 212301 (2002).

[2] C. Adler et al. (STAR Collaboration), Phys. Rev. Lett. 90, 032301 (2003); S. S. Adler et al. (PHENIX Collaboration), ibid. 91, 182301 (2003); J. Adams et al. (STAR Collaboration), ibid. 92, 052302 (2004).

[3] P. F. Kolb, J. Sollfrank, and U. Heinz, Phys. Rev. C 62, 054909 (2000); P. F. Kolb, P. Huovinen, U. Heinz, and H. Heiselberg, Phys. Lett. B500, 232 (2001); P. Huovinen, P. F. Kolb, U. Heinz, P. V. Ruuskanen, and S. A. Voloshin, Phys. Lett. B503, 58 (2001).

[4] D. Teaney, J. Lauret, and E. V. Shuryak, Phys. Rev. Lett. 86, 4783 (2001); and nucl-th/0110037.

[5] X. N. Wang, Phys. Rev. C 63, 054902 (2001); M. Gyulassy, I. Vitev, and X. N. Wang, Phys. Rev. Lett. 86, 2537 (2001).

[6] K. Adcox et al. (PHENIX Collaboration), Phys. Rev. Lett. 88, 022301 (2002); J. Adams et al. (STAR Collaboration), ibid. 91, 172302 (2003).

[7] S. Turbide, R. Rapp, and C. Gale, Phys. Rev. C 69, 014903 (2004).

[8] S. Turbide, C. Gale, S. Jeon, and G. D. Moore, Phys. Rev. C 72, 014906 (2005).

[9] S. A. Bass, B. Müller, and D. K. Srivastava, Phys. Rev. Lett. 90, 082301 (2003); Phys. Rev. C 66, 061902(R) (2002); T. Renk, S. A. Bass, and D. K. Srivastava, Phys. Lett. B632, 632 (2006).

[10] R. J. Fries, B. Müller, and D. K. Srivastava, Phys. Rev. Lett. 90, 132301 (2003); Phys. Rev. C 72, 041902(R) (2005).

[11] S. S. Adler et al. (PHENIX Collaboration), Phys. Rev. Lett. 94, 232301 (2005); Eur. Phys. J. C 43, 421 (2005).

[12] U. Heinz and P. F. Kolb, Nucl. Phys. A702, 269 (2002); U. Heinz, ibid. A721, 30 (2003); M. Gyulassy, in Structure and Dynamics of Elementary Matter, edited by W. Greiner et al., Nato Science Series II, Vol. 166 (Kluwer Academic, Dordrecht, 2004), p. 159;M. Gyulassy and L. McLerran, Nucl. Phys. A750, 30 (2005); E. V. Shuryak, ibid. A750, 64 (2005).

[13] T. Hirano, Phys. Rev. C 65, 011901(R) (2001); T. Hirano and K. Tsuda, ibid. 66, 054905 (2002).

[14] P. F. Kolb and U. Heinz, in Quark Gluon Plasma 3, edited by R. C. Hwa and X. N. Wang (World Scientific, Singapore, 2004), p. 634.

[15] P. Huovinen, in Quark Gluon Plasma 3, edited by R. C. Hwa and X. N. Wang (World Scientific, Singapore, 2004), p. 600;
P. F. Kolb and U. Heinz, ibid., p. 634; E. V. Shuryak, Prog. Part. Nucl. Phys. 53, 273 (2004); P. Huovinen and P. V. Ruuskanen, Annu. Rev. Nucl. Part. Sci. 56, 163 (2006).

[16] T. Hirano, U. Heinz, D. Kharzeev, R. Lacey, and Y. Nara, Phys. Lett. B636, 299 (2006).

[17] P. Huovinen, Nucl. Phys. A761, 296 (2005).

[18] T. Lappi and R. Venugopalan, Phys. Rev. C 74, 054905 (2006).

[19] J. I. Kapusta and C. Gale, Finite-Temperature Field Theory: Principles and Applications (Cambridge University Press, Cambridge, 2006).

[20] P. V. Ruuskanen, in Particle Production in Highly Excited Matter, edited by H. H. Gutbrod and J. Rafelski, NATO Adv. Study Inst. Ser. B Phys. 303, 593 (Plenum, New York, 1993).

[21] H. Sorge, Phys. Rev. Lett. 78, 2309 (1997); 82, 2048 (1999).

[22] S. Turbide, C. Gale, and R. J. Fries, Phys. Rev. Lett. 96, 032303 (2006).

[23] R. Chatterjee, E. S. Frodermann, U. Heinz, and D. K. Srivastava, Phys. Rev. Lett. 96, 202302 (2006).

[24] K. Geiger and J. I. Kapusta, Phys. Rev. Lett. 70, 1920 (1993).

[25] E. L. Feinberg, Nuovo Cimento A 34, 391 (1976).

[26] E. V. Shuryak, Phys. Lett. B78, 150 (1978).

[27] L. D. McLerran and T. Toimela, Phys. Rev. D 31, 545 (1985).

[28] R. C. Hwa and K. Kajantie, Phys. Rev. D 32, 1109 (1985).

[29] J. Kapusta, L. McLerran, and D. K. Srivastava, Phys. Lett. B283, 145 (1992).

[30] T. Altherr and P. V. Ruuskanen, Nucl. Phys. B380, 377 (1992).

[31] M. H. Thoma and C. T. Traxler, Phys. Rev. D 56, 198 (1997).

[32] D. K. Srivastava, M. G. Mustafa, and B. Müller, Phys. Rev. C 56, 1064 (1997).

[33] K. Kajantie, J. Kapusta, L. McLerran, and A. Mekjian, Phys. Rev. D 34, 2746 (1986)

[34] K. Kajantie, M. Kataja, L. McLerran, and P. V. Ruuskanen, Phys. Rev. D 34, 811 (1986).

[35] C. Gale and J. I. Kapusta, Nucl. Phys. B357, 65 (1991).

[36] J. Cleymans, K. Redlich, and H. Satz, Z. Phys. C 52, 517 (1991); J. Cleymans, V. V. Goloviznin, and K. Redlich, Phys. Rev. D 47, 173 (1993); D. Pal, K. Haglin, and D. K. Srivastava, Phys. Rev. C 54, 1366 (1996); D. Pal, P. K. Roy, S. Sarkar, D. K. Srivastava, and B. Sinha, ibid. 55, 1467 (1997).

[37] R. Rapp, C. Chanfray, and J. Wambach, Nucl. Phys. A617, 472 (1997); R. Rapp, Phys. Rev. C 63, 054907 (2001). 
[38] I. Kvasnikova, C. Gale, and D. K. Srivastava, Phys. Rev. C 65, 064903 (2002).

[39] S. Damjanovic et al. (NA60 Collaboration), Nucl. Phys. A774, 715 (2006).

[40] See, e.g., M. G. Mustafa, D. Pal, D. K. Srivastava, and M. Thoma, Phys. Lett. B428, 889 (1998); I. P. Lokhtin and A. M. Snigirev, Eur. Phys. J. C 21, 155 (2001); K. Gallmeister, B. Kämpfer, and O. P. Pavlenko, Nucl. Phys. A715, 705 (2003); B. Zhang, L.-W. Chen, and C. M. Ko, Phys. Rev. C 72, 024906 (2005); Z. W. Lin and D. Molnar, ibid. 68, 044901 (2003); F. Laue et al. (STAR Collaboration), J. Phys. G 31, S1121 (2005).

[41] J. D. Bjorken, Phys. Rev. D 27, 140 (1983).

[42] The code can be downloaded from URL http://nt3.phys. columbia.edu/people/molnard/OSCAR/. See also the first paper in [3], and P. F. Kolb and R. Rapp, Phys. Rev. C 67, 044903 (2003).

[43] D. Seibert and T. Altherr, Phys. Rev. D 48, 3386 (1993).

[44] A. J. Kuhlman and U. Heinz, Phys. Rev. C 72, 037901 (2005).

[45] S. Turbide, C. Gale, D. K. Srivastava, and R. J. Fries, Phys. Rev. C 74, 014903 (2006).

[46] S. S. Adler et al. (PHENIX Collaboration), Phys. Rev. C 72, 014903 (2005).

[47] For hadrons it is known that the hydrodynamically predicted monotonic rise of $v_{2}\left(p_{T}\right)$ is broken by viscous effects at large transverse momenta, leading instead to a saturation of $v_{2}$ for $p_{T} \geqslant 1.5-2 \mathrm{GeV} / c$ for mesons and for $p_{T} \geqslant 2-2.5 \mathrm{GeV} / c$ for baryons [48]. Within the successful quark coalescence model for intermediate- $p_{T}$ hadron production [49] this can be interpreted as quark elliptic flow following the hydrodynamic prediction to $p_{T} \simeq 0.75-0.8 \mathrm{GeV} / c$, followed by saturation at larger transverse momenta [48]. Becuase dileptons with large invariant mass $M$ and/or large $p_{T}$ are formed in collisions involving high- $p_{T}$ quarks and antiquarks it is likely that the same viscous effects also break the hydrodynamically predicted monotonic rise of the hadronic dilepton elliptic flow seen in all results presented here. Similarly, viscous effects are expected to further reduce the elliptic flow of quark matter dileptons with large invariant masses and/or $p_{T}$, below the alredy small values shown here. A quantitative assessment of this issue requires hydrodynamic simulations for viscous fluids and will have to await future studies.

[48] U. Heinz, AIP Conf. Proc. 739, 163 (2004).

[49] V. Greco, C. M. Ko, and P. Levai, Phys. Rev. Lett. 90, 202302 (2003); R. J. Fries, B. Müller, C. Nonaka, and S. A. Bass, ibid. 90, 202303 (2003); D. Molnar and S. A. Voloshin, ibid. 91, 092301 (2003).

[50] Homogeneity regions are regions of space-time contributing with roughly constant probability to the emission of particles with a given fixed momentum.

[51] S. Voloshin, preprint TPI-MINN-90-33-T (1990, unpublished).

[52] J. Y. Ollitrault, Phys. Rev. D 46, 229 (1992); R. S. Bhalerao, J. P. Blaizot, N. Borghini, and J. Y. Ollitrault, Phys. Lett. B627, 49 (2005).

[53] R. Rapp, Phys. Rev. C 63, 054907 (2001). 